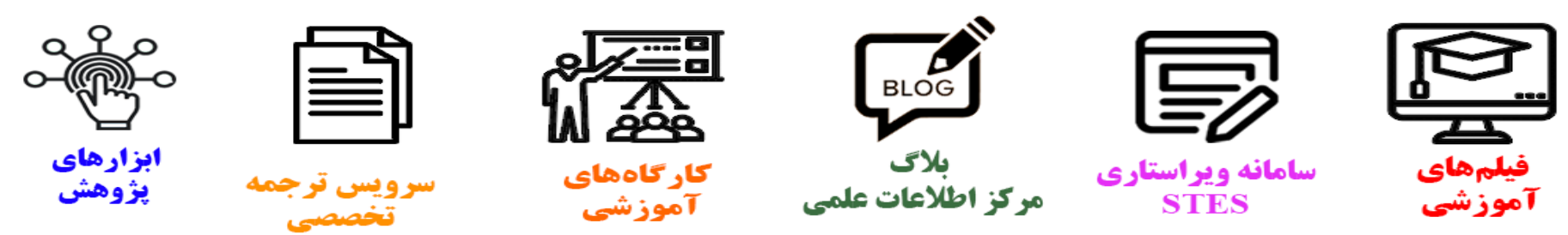

\title{
(c)
}

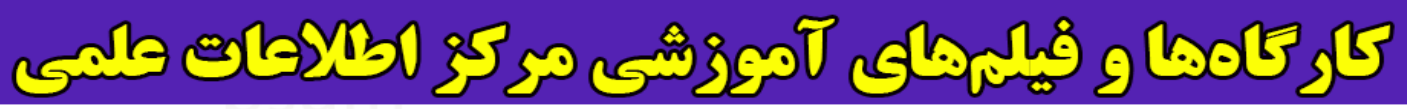
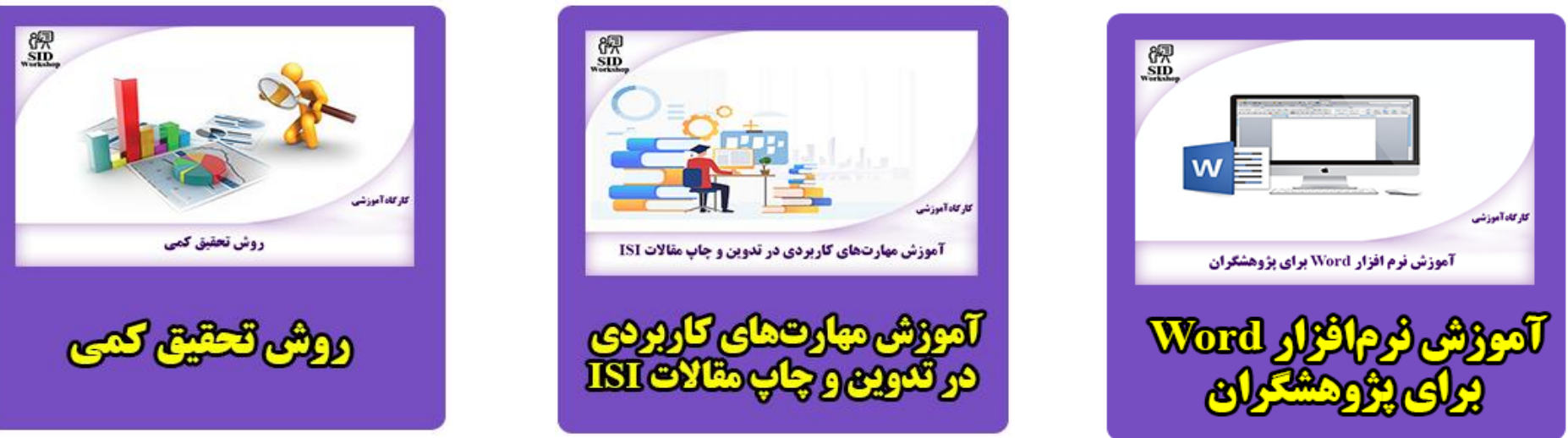


\title{
RESEARCH NOTE
}

\section{NONLINEAR SENSITIVITY ANALYSIS OF RCMRF CONSIDERING BOTH AXIAL AND FLEXURE EFFECTS}

\author{
A.R. Habibi \\ Department of Civil Engineering, University of Kurdistan \\ P.O. Box 416, Sanandaj, Iran \\ ar.habibi@uok.ac.ir
}

(Received: February 07, 2010 - Accepted in Revised Form: September 15, 2011)

doi: 10.5829/idosi.ije.2011.24.03a.02

\begin{abstract}
Sensitivity analysis of structures considering their nonlinear behavior has recently been an important process in optimal design of them based on new design concepts such as PerformanceBased Design (PBD). The main objective of this research is to develop an efficient and applicable method for nonlinear sensitivity analysis of Reinforcement Concrete Moment Resisting Frames (RCMRF) considering both axial and flexure effects under seismic loading. For this purpose, sensitivity equations are firstly derived based on pushover method, which is a powerful tool for nonlinear analysis of structures in the PBD context, and then a procedure for nonlinear sensitivity analysis is proposed. The results of the method are compared with nonlinear sensitivity results considering only flexural effect and those of Finite Difference Method (FDM). It is shown that existence of axial forces can affect sensitivity coefficients with respect to the design variables.
\end{abstract}

Keywords Nonlinear, Sensitivity, Analysis, Pushover, RCMRF, PBD, Axial and Flexure Effects, Seismi

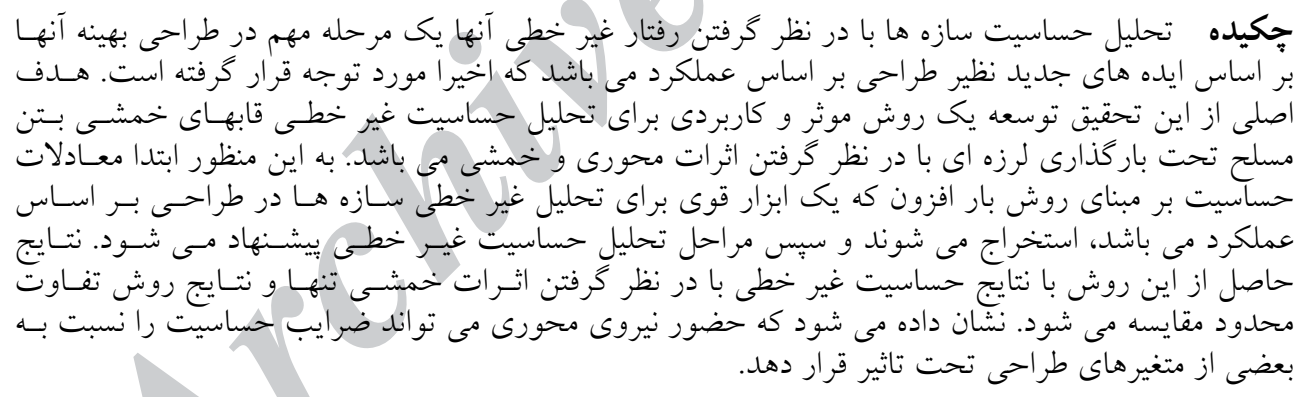

\section{INTRODUCTION}

Considerable part of computational effort in an optimization problem is usually allocated to the sensitivity analysis. Each sensitivity coefficient defines the amount of change in a structural response due to a unit change in a design variable, such as sensitivity of displacement to change in cross-sectional dimensions or reinforcement ratio. In recent years, new design concepts such as performance-based design have become a necessity for design of new structures. Since nonlinear methods such as pushover analysis are used in these new design concepts, it is necessary that efficient nonlinear sensitivity methods be employed in optimum design.

While considerable research effort has been put on developing the nonlinear sensitivity analysis techniques, there are a few research works in the literature that have focused on the theory of sensitivity analysis for nonlinear-framed structures. Ryu et al. proposed a general nonlinear sensitivity analysis considering geometric and material nonlinearity [1]. They presented the formulation 
for a truss example. Choi and Santos developed variational formulations for nonlinear design sensitivity analysis [2]. Gopalakrishna and Greimann differentiated the equilibrium equation in each Newton-Raphson iteration to obtain incremental gradients and this method was used for the nonlinear sensitivity analysis of the plane trusses [3]. Santos and Choi presented a unified approach for shape sensitivity analysis of trusses and beams considering both geometric and material nonlinearities [4]. Ohsaki and Arora presented an accumulative and incremental algorithm for the design sensitivity analysis of elastoplastic structures including geometrical nonlinearity [5]. They performed the sensitivity analysis of trusses but they reported that the method is extremely time consuming for large structures. Lee and Arora developed design sensitivity analysis of structural systems with elastoplastic material behavior using the continuum formulation and performed sensitivity analysis of a truss and a plate by this technique [6]. Barthold and Stein presented a continuum mechanical-based formulation for the variational sensitivity analysis considering nonlinear hyper elastic material behavior using either the Lagrangian or Eulerian description [7]. Szewczyk and Ahmed presented a hybrid numerical/neurocomputing strategy for evaluation of sensitivity coefficients of composite panels subjected to combined thermal and mechanical loads [8]. Yamazaki suggested a direct sensitivity analysis technique for finding incremental sensitivities of the path-dependent nonlinear problem based on the updated Lagrangian formulation [9]. Employing this method, they performed the sensitivity analysis of a plate. Bugeda et al. proposed a direct formulation for computing the structural shape sensitivity analysis with a nonlinear constitutive material model [10]. It was reported that their proposed approach was valid for some specific nonlinear material models. Schwarz and Ramm proposed the variational direct method for sensitivity analysis of structural response considering geometrical and material nonlinearity with Prantel-Reuss plasticity model [11]. Gong et al. presented a procedure for sensitivity analysis of planar steel moment frameworks with geometric and material nonlinearity [12]. In their work, analytical formulations defining the sensitivity of displacement were derived. Habibi A.R. and Moharrami H. developed a comprehensive formulation for sensitivity analysis of planar RCMRFs considering material nonlinearity and P$\Delta$ effects under pushover analysis based on Newton-Raphson method [13]. In their research, only flexure effect was considered to derive sensitivity equations.

The objective of present research is to develop the nonlinear theory of sensitivity analysis for RC frames considering both axial and flexure effects. Also, sensitivity equations are modified to conclude effect of seismic loading that depends on design variables. To do this, pushover analysis that is a simplified nonlinear analysis is employed. The sensitivity equations are derived based on the analysis with incremental-load form. A three-story RCMRF has been used as an example to illustrate the applicability and efficiency of the developed sensitivity formulations.

\section{NONLINEAR ANALYSIS}

The first step of design optimization is the sensitivity analysis that in turn depends on the method of structural analysis. The simplest recommended method for nonlinear analysis is pushover method which is used in this study. This method of analysis that is recommended by FEMA273 [14] and ATC40 [15] is a popular tool for evaluation of seismic performance of existing and new structures. A proper material behavior model for elements and seismic loads for pushover analysis are explained in the next three sections.

2.1. Moment Curvature Relation The momentcurvature relation of every Reinforced Concrete (RC) structural element has a definitive effect on the behavior of the structure. In this research the tri-linear moment curvature relation, as shown in Figure 1, is used for expressing the nonlinear behavior of reinforced concrete sections.

To determine moment-curvature relation of $\mathrm{RC}$ members, some assumptions should be made that best fits with the test results. In this study, considering the effect of axial force in momentcurvature relation, the following limitations for 


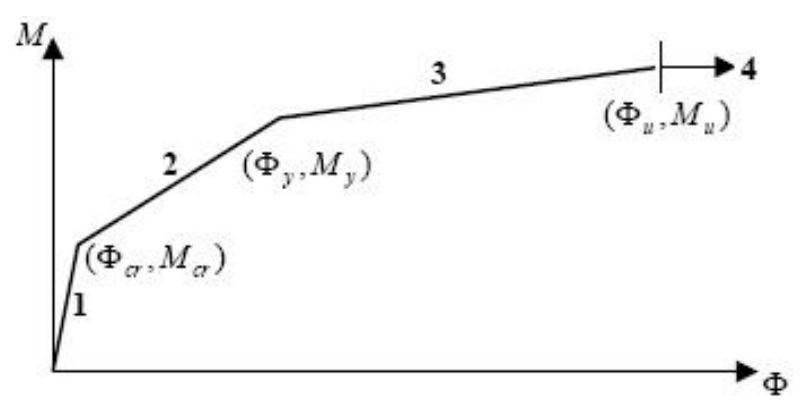

Figure 1. Tri-linear moment curvature curve.

various states of behavior of RC elements are used [16,17]:

(a) Cracking state

$$
\begin{aligned}
& M_{c r}=f_{r} I /(h-y)+N d / 6 \\
& \phi_{c r}=f_{r} / E_{c}(h-y)
\end{aligned}
$$

(b) Yielding state

$$
M_{y}=0.5 f_{c} b_{t} d^{2}\left[(1+\beta-\eta) n_{0}+(2-\eta) p+(\eta-2 \beta) \alpha p^{\prime}\right]
$$

$\varphi_{y}=c \varepsilon_{y} /(1-k) d$

(c) Ultimate state

$$
\begin{aligned}
& M_{u}=\left(1.24-0.15 p-0.5 n_{0}\right) M_{y} \\
& \varphi_{u}=1.05 \beta_{1} \varepsilon_{u} /\left[\left(R^{2}+S\right)^{0.5}-R\right]
\end{aligned}
$$

where,

$R=\left(\rho^{\prime} \varepsilon_{u} E_{s}-\rho f_{y}\right) d /\left(1.7 f_{c}\right) ; S=\rho^{\prime} \varepsilon_{u} E_{s} \beta_{1} d^{\prime} d /\left(0.85 f_{c}\right)$;

$f_{r}$ is the modulus of rupture of concrete; $f_{c}$ is the cylinder strength of concrete; $f_{y}$ is the yield strength of steel; $E_{c}$ is the modulus of elasticity of concrete; $E_{s}$ is the modulus of elasticity of steel; $\varepsilon_{u}$ is the ultimate strain of concrete; $\varepsilon_{y}$ is the yield strain of steel; $\beta_{1}$ is a coefficient that depends on the strength of concrete; $\mathrm{N}$ is the axial force; $d$ is the depth to rebar; $\lambda^{\prime}{ }^{\prime}$ is the cover depth for compression bars; $h$ is the overall height of section; $\mathrm{c}$ is the cover to steel centroid; $b_{t}$ is the top width of section; $y$ is the distance from the neutral axis of the section to the extreme fiber in tension and is obtained from (7); I is the moment of inertia of the section and is obtained from Equation 8. Other parameters are defined from Equations 9 to 16.

$$
\begin{aligned}
& y=\frac{0.5 b_{t} t^{2}+0.5 b_{b}\left(h^{2}-t^{2}\right)+\left(n_{s c}-1\right)\left[A_{s}^{\prime} d^{\prime}+A_{s} d\right]}{\left[b_{t} t+b_{b}(h-t)+\left(n_{s c}-1\right)\left(A_{s}+A_{s}^{\prime}\right)\right]} \\
& I=b_{t} t^{3} / 12+b_{t} t(y-0.5 t)^{2}+b_{b}(h-t)^{3} / 12+b_{b}(h-t) \times \\
& (0.5 h+0.5 t-y)^{2}+\left(n_{s c}-1\right) \times\left[A_{s}(d-y)^{2}+A_{s}^{\prime}\left(y-d^{\prime}\right)^{2}\right]
\end{aligned}
$$

$$
k=\left\{\begin{array}{lr}
\sqrt{\left(p+p^{\prime}\right)^{2} \frac{1}{4 \alpha^{2}}+\left(p+\beta p^{\prime}\right) \frac{1}{\alpha}}-\left(p+p^{\prime}\right) \frac{1}{2 a} & k \leq \frac{t}{d} \\
\sqrt{\left[n_{s c}\left(\rho+\rho^{\prime}\right)+\frac{t}{d}\left(\frac{b_{t}}{b_{b}}-1\right)\right]^{2}+2 n_{s c}\left(\rho+\rho^{\prime} \beta\right)+\frac{t^{2}}{d^{2}}\left(\frac{b_{t}}{b_{b}}-1\right)} & k>\frac{t}{d} \\
-n_{s c}\left(\rho+\rho^{\prime}\right)-\frac{t}{d}\left(\frac{b_{t}}{b_{b}}-1\right) &
\end{array}\right.
$$

$\eta=\frac{0.75}{1+\varepsilon_{y} / \varepsilon_{0}}\left(\frac{\varphi_{y} d-\varepsilon_{y}}{\varepsilon_{0}}\right)^{0.7}$

$\alpha=(1-\beta)\left(\frac{\varphi_{y} d-\varepsilon_{y}}{\varepsilon_{y}}\right)-\beta \leq 1$

$\rho=A_{s} / b_{b} d ; \rho^{\prime}=A_{s}^{\prime} / b_{b} d$

$p^{\prime}=\rho^{\prime} f_{y} / f_{c} ; p=\rho f_{y} / f_{c}$

$\beta=d^{\prime} / d$

$n_{0}=N / f_{c} b d$

$c=1.05+\left(\frac{0.45}{0.84+p}-0.05\right) \frac{n_{0}}{0.3}$

where $n_{s c}$ is the ratio of the modulus of elasticity of steel to that of concrete; $\varepsilon_{0}$ is the strain at maximum strength of concrete; $b_{b}$ is the width of the section at the bottom; $t$ is the flange thickness of $\mathrm{T}$ or $\mathrm{L}$ beam; $A_{s}$ is the area of bottom bars and $A_{s}^{\prime}$ is the area of top bars. For the case of positive moment, Equations 1 to 16 are valid for all types of sections. For rectangular sections these equations can be simplified by substituting $b_{b}=b_{t}=b$ and $t=0$. Also, for the case of negative 
moment, these equations can be used by substituting $b_{t}, b_{b}, h-t, A_{s}^{\prime}$ and $A_{s}$ instead of $b_{b}$, $b_{t}, t, A_{s}$ and $A_{s}^{\prime}$, respectively. Ignoring axial effect in beam elements, $\mathrm{N}$, which has been appeared in some above equations, can be considered to be equal to zero. Now by having the moment-curvature relations, the flexural stiffness can be specified for ends of the element as follow:

$$
\begin{aligned}
& E I_{p}=M_{c r p} / \varphi_{c r p} \leq E I_{p m} \\
& E I_{p}=\left(M_{y p}-M_{c r p}\right) /\left(\varphi_{y p}-\varphi_{c r p}\right) \leq E I_{p m} \\
& E I_{p}=\left(M_{u p}-M_{y p}\right) /\left(\varphi_{u p}-\varphi_{y p}\right) \leq E I_{p m}
\end{aligned}
$$

where Equations 17-19 are used for zones of 1, 2 and 3 of $M-\phi$ curve, respectively. In these equations, $E I_{p}$ and $E I_{p m}$ are the stiffness and minimum stiffness of any section; $M_{c r p}, M_{y p}$ and $M_{u p}$ are the cracking, yielding and ultimate moments; and $\phi_{c r p}, \phi_{y p}$ and $\phi_{u p}$ are corresponding curvatures.

2.2. Material Nonlinearity In this paper, a spread plasticity model that has been proposed by park et al. is utilized [18]. This plasticity model, that considers the cracking behavior of $\mathrm{RC}$, can also consider material nonlinearity of RC elements with a very good approximation. It has been implemented in IDARK software [18]. In this model, for an element subjected to earthquake loading, the distribution of curvature follows Figures $2 \mathrm{a}, \mathrm{b}$ which is considered for the flexibility distribution in the RC elements, where $E I_{A}$ and $E I_{B}$ are the flexural stiffness of the section at end 'A' and 'B', respectively, and $E I_{0}$ is the initial stiffness of the element; $\alpha_{A}$ and $\alpha_{B}$ are the yield penetration coefficients and $L^{\prime}$ is the length of the element.

Adopting the above plasticity model for RC elements, the tangent stiffness matrix of each element can be derived. General derivation of tangent stiffness matrix is given by Park et al. [18]. Considering a RC element with six degrees of freedom, with its rigid parts, as shown in Figure 3, the tangent stiffness matrix of element can be

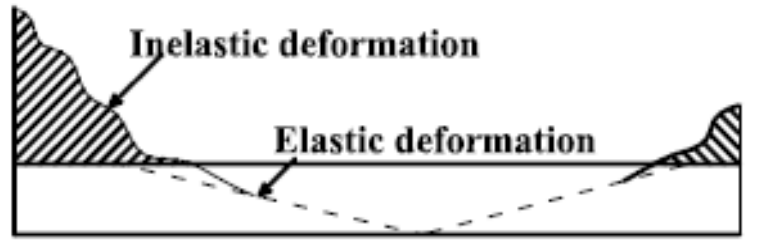

(a)

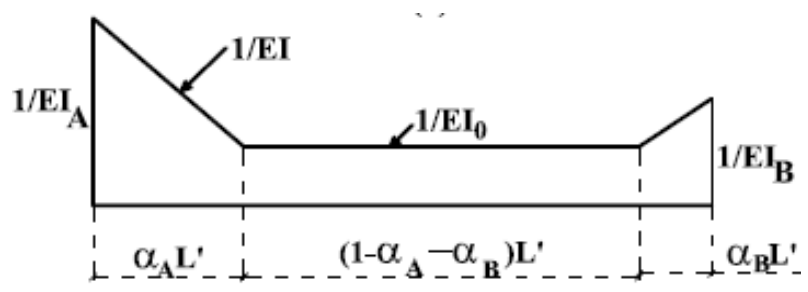

(b)

Figure 2. (a) Curvature distribution along a RC element and (b) Flexibility assumption along a RC element.

found as follows:

$K_{t e}=K_{a}+K_{b}$

where $K_{a}$ and $K_{b}$ are axial stiffness matrix and bending stiffness matrix, respectively and must be assembled in the element stiffness matrix $K_{t e}$. The element stiffness matrix calculated from Equation 20 needs to be transformed to global system:

$K_{e}=T_{e}^{T} K_{t e} T_{e}$

where $T_{e}$ is the transformation matrix of the element.

$K_{a}$ can be determined from:

$K_{a}=\left[\begin{array}{cc}1 & -1 \\ -1 & 1\end{array}\right] \frac{E A}{L}=A K_{a 0}$

where $E A / L$ is the axial stiffness of the element, $A$ is the area of cross section and $K_{a 0}$ is a constant matrix. $K_{b}$, is obtained from the following equation:

$K_{b}=R_{E} K_{S} R_{E}^{T}$ 


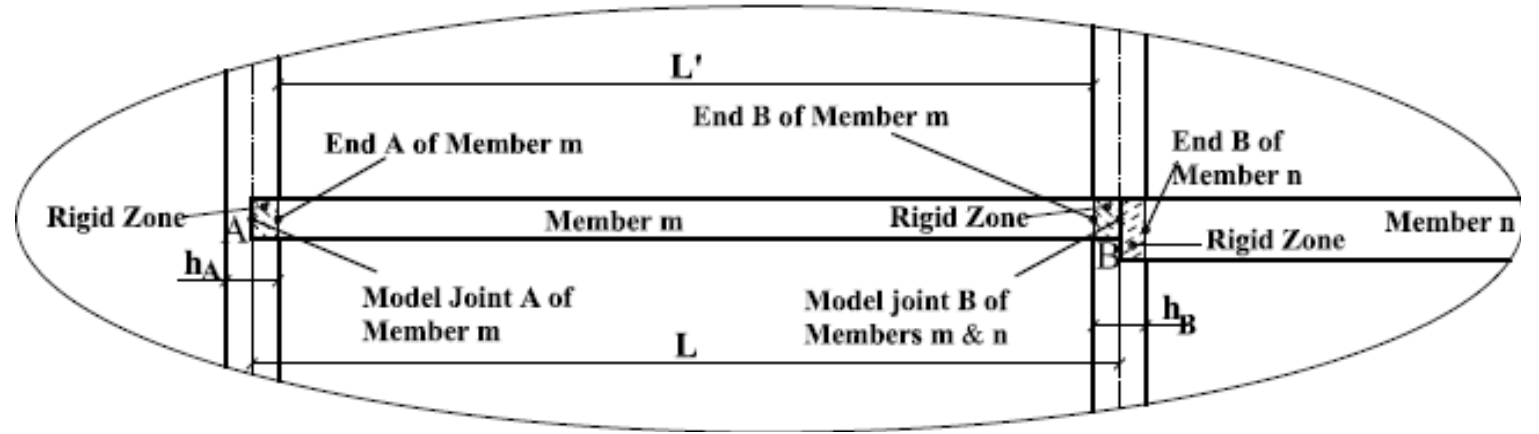

Figure 3. Rigid zone and ends definitions.

where:

$R_{E}^{T}=\left[\begin{array}{cccc}-1 / L & 1 & 1 / L & 0 \\ -1 / L & 0 & 1 / L & 1\end{array}\right] ; K_{S}=\tilde{L} K_{S}^{\prime} \tilde{L}^{-1}$

where $L$ is length of element and $\tilde{L}$ can be obtained from the following equation:

$$
\tilde{L}=\frac{1}{1-\lambda_{A}-\lambda_{B}}\left[\begin{array}{cc}
1-\lambda_{B} & \lambda_{A} \\
\lambda_{B} & 1-\lambda_{A}
\end{array}\right] ; \tilde{L}^{-1}=\left[\begin{array}{cc}
1-\lambda_{A} & -\lambda_{A} \\
-\lambda_{B} & 1-\lambda_{B}
\end{array}\right]
$$

where $\lambda_{A}$ and $\lambda_{B}$ are the portions of rigid zone at the element ends. The elements of $K_{S}^{\prime}$ in (24) are obtained from the following equation:

$$
\left.\begin{array}{l}
K_{S 11}=12 E I_{0} E I_{A} E I_{B} L^{\prime} f_{b} / D_{e} \\
K_{S 12}=K_{S 21}=K_{S 11} f_{c} / f_{b} ; K_{S 22}=K_{S 11} f_{a} / f_{b}
\end{array}\right\}
$$

where:

$$
\left.\begin{array}{l}
f_{a}=4 S_{1}+S_{2}\left(6 \alpha_{A}-4 \alpha_{A}^{2}+\alpha_{A}^{3}\right)+S_{3} \alpha_{B}^{3} \\
f_{b}=4 S_{1}+S_{2} \alpha_{A}^{3}+S_{3}\left(6 \alpha_{B}-4 \alpha_{B}^{2}+\alpha_{B}^{3}\right) \\
f_{c}=-2 S_{1}-S_{2}\left(2 \alpha_{A}^{2}-\alpha_{A}^{3}\right)-S_{3}\left(2 \alpha_{B}^{2}-\alpha_{B}^{3}\right) \\
D_{e}=\left(f_{a} f_{b}-f_{c}^{2}\right) L^{\prime 2} ; L^{\prime}=\left(1-\lambda_{A}-\lambda_{B}\right) L
\end{array}\right\}
$$

where

$$
\begin{aligned}
& S_{1}=E I_{A} E I_{B} ; \quad S_{2}=\left(E I_{0}-E I_{A}\right) E I_{B} ; \quad \text { and } \\
& S_{3}=\left(E I_{0}-E I_{B}\right) E I_{A} .
\end{aligned}
$$

The yield penetration parameters used in Equation 27 , specify the portion of the element where it cracks. When the moment of the section under consideration is less than cracking moment, the values of these parameters are considered to be equal to zero. For the single curvature of the element, when its moments are greater than cracking moment, the values of the parameters are considered to be equal to 0.5. For other cases, assuming linear moment distribution along the element, these parameters can be determined from:

$\alpha_{p}=\max \left\{\min \left\{\left(\left|M_{p}\right|-M_{c r p}\right) /\left|M_{A}-M_{B}\right|, 1\right\}, \alpha_{p m}\right\}$

where subscript $\mathrm{p}$ specifies any general point $\mathrm{p}$ and cr represents the cracking state under consideration. $\alpha_{p m}$ is the maximum yield penetration parameter, obtained in previous load steps. Usually, the flexural stiffness at the center of the element is equal to elastic stiffness and is determined from Equation 29. For the case of single curvature if moments are greater than cracking moments, their values can be modified by Equation 30.

$$
\begin{aligned}
& E I_{0}=\frac{2 E I_{A 0} E I_{B 0}}{E I_{A 0}+E I_{B 0}} \\
& E I_{0}=\frac{2 E I_{A} E I_{B}}{E I_{A}+E I_{B}}
\end{aligned}
$$

where $E I_{A 0}$ and $E I_{B 0}$ are the elastic stiffness at ends 'A' and 'B', respectively; and $E I_{A}$ and $E I_{B}$ 
are the inelastic stiffness at ends ' $A$ ' and ' $B$ ', respectively. For a member that yield penetration spreads over the whole element (when $\alpha_{A}+\alpha_{B} \geq 1$ ), $E I_{0}$ is obtained from Equation 30. In this case, $\alpha_{A}$ and $\alpha_{B}$ and initial flexibility are modified to capture the actual flexibility distribution. These modifications are:

$$
\alpha_{A}^{\prime}=\frac{f_{A}-f_{B}+\frac{f_{B}-f_{0}}{\alpha_{B}}}{\frac{f_{A}-f_{0}}{\alpha_{A}}+\frac{f_{B}-f_{0}}{\alpha_{B}}}
$$

$\alpha_{B}^{\prime}=1-\alpha_{A}^{\prime}$

$f_{0}^{\prime}=f_{A}-\left(f_{A}-f_{0}\right) \alpha_{A}^{\prime} / \alpha_{A}$

where:

$$
f_{A}=1 / E I_{A} ; f_{B}=1 / E I_{B} ; f_{0}=1 / E I_{0}
$$

2.3. Seismic Loading In the pushover analysis, the load vector must incrementally be increased. At each load step, the base shear increment is applied to the structure with a predefined profile over the height of the structure. The incremental lateral load vector can be computed as:

$\Delta P_{E}=\Delta V_{b} \cdot\left\{\begin{array}{c}c_{v, 1} \\ c_{v, 2} \\ \vdots \\ c_{v, n s}\end{array}\right\}=\Delta V_{b} \cdot C_{v}$

where $\Delta V_{b}$ is the incremental base shear and $C_{v}$ is the vector of lateral load distribution factors $c_{v, s}(s=1, \ldots$, number of stories $)$, which is determined using the following relation [14]:

$$
c_{v, s}=\frac{W_{s} H_{s}^{k}}{\sum_{s=1}^{n s} W_{s} H_{s}^{k}}, s=1, \ldots, n s
$$

where $W_{s}$ is the portion of the building seismic weight at story level $s ; H_{s}$ is the vertical distance from base of the building to story level $s ; n s$ is the number of stories; and $k$ is a parameter that has been recommended by FEMA273 as follows [14]:

$$
k= \begin{cases}1 & T<0.5 \\ 0.5 T+0.75 & 0.5 \leq T \leq 2.5 \\ 2 & T>2.5\end{cases}
$$

where $T$ is the fundamental period of the building.

2.4. Nonlinear Responses Since in nonlinear analysis of structure, stiffness isn't constant and depends on the internal forces of elements and internal forces depend on displacement field, it will be a function of displacement field; on the other hand, displacement depends on stiffness. Then structural analysis is involved in incremental process. The equilibrium equation of the structure is solved using the simple incremental method considering several load steps. No iteration is required within one load step, and thus the numerical algorithm is generally well behaved and exhibits good computational efficiency. In each load step following equation must be solved [19]:

$$
K_{T}^{l} \Delta u^{l}=\Delta P^{l}
$$

where $K_{T}^{l}$ is the tangent stiffness of the structure at load step $l ; \Delta u^{l}$ is the incremental displacement vector of the structure at load step $l$ and $\Delta P^{l}$ is the external load vector imposed on the structure at load step $l$. By having the incremental displacement vector from Equation 38, the displacement vector of the structure at each load step must be updated as follows:

$u^{l}=u^{l-1}+\Delta u^{l}$

where $u^{l-1}$ and $u^{l}$ are the displacement vector of the structure at load steps $(l-1)$ and $(l)$, respectively. Considering equilibrium of forces for an element, the incremental internal forces of the element at load step $l$ can be determined from Equation 40:

$\Delta F_{e}^{l}=K_{e}^{l} \Delta u_{e}^{l}$

where $K_{e}^{l}$ is the tangent stiffness matrix of the 
element and $\Delta u_{e}^{l}$ is the incremental displacement vector of the element at load step $l$. By having the incremental internal forces from last mentioned equation, the internal forces of the element at load step $l$ must be updated as follows:

$F_{e}^{l}=F_{e}^{l-1}+\Delta F_{e}^{l}$

where $F_{e}^{1}$ and $F_{e}^{\prime}$ are the internal force vectors of the element at load steps $(l-1)$ and $(l)$, respectively.

\section{NONLINEAR SENSITIVITY ANALYSIS}

In the structures undergoing inelastic behavior, incremental methods are usually used to solve equilibrium equation. In this case, displacements are nonlinear function of external loads. Also stiffness is a function of displacements and forces. This causes sensitivity coefficients such as displacement sensitivities to be dependent on stiffness sensitivity, displacement sensitivity, external load sensitivity and internal load sensitivity; while in linear structures, they only depend on stiffness sensitivity and external load sensitivity. Finding sensitivity relations in nonlinear structures is too difficult for this complicacy; on the other hand, applying linear sensitivity theory leads to incorrect results. In the present study, to drive relations for nonlinear sensitivities, equilibrium equations are differentiated at each load step. This is explained in the next section.

\subsection{Displacement Sensitivity Considering} incremental Equation 38 and differentiating it with respect to design variable $x_{j}$, we get to:

$$
\frac{d K_{T}^{l}}{d x_{j}} \Delta u^{l}+K_{T}^{l} \frac{d \Delta u^{l}}{d x_{j}}=\frac{d \Delta P^{l}}{d x_{j}}
$$

where $K_{T}^{l}$ is the global tangent stiffness matrix; $\Delta u^{l}$ is the incremental displacement vector; $\frac{d K_{T}^{l}}{d x_{j}}$ is the sensitivity of the global tangent stiffness matrix; $\frac{d \Delta u^{l}}{d x_{j}}$ is the sensitivity of the incremental displacement vector and $\frac{d \Delta P^{l}}{d x_{j}}$ is the sensitivity of the incremental external load vector at load step $l$ with respect to design variable $x_{j}$. In this study, Equation 42 is used as the basic formulation for obtaining the incremental displacement sensitivities. Assuming that all terms in Equation 42 except $\frac{d \Delta u^{l}}{d x_{j}}$ are known, it will be rearranged to find:

$$
\frac{d \Delta u^{l}}{d x_{j}}=\left[K_{T}^{l}\right]^{-1}\left(\frac{d \Delta P^{l}}{d x_{j}}-\frac{d K_{T}^{l}}{d x_{j}} \Delta u^{l}\right)
$$

The total sensitivity of displacement vector can be obtained by summing up the sensitivities in all load steps as follows:

$$
\frac{d u^{l}}{d x_{j}}=\frac{d u^{l-1}}{d x_{j}}+\frac{d \Delta u^{l}}{d x_{j}}
$$

where $\frac{d u^{l-1}}{d x_{j}}$ and $\frac{d u^{l}}{d x_{j}}$ are the sensitivities of the incremental displacement vector at load steps $(l-1)$ and $(l)$, respectively. The term $\frac{d \Delta u^{l}}{d x_{j}}$ is the sensitivity of the incremental displacement vector which is obtained from Equation 43. For this, sensitivities of stiffness and external loads need to be determined. In the coming sections, the way each sensitivity item in Equation 43 should be obtained, is explained.

3.1.1. Sensitivity of stiffness The tangent stiffness matrix of the structure at each load step is obtained by properly assembling of the tangent stiffness matrix of elements:

$$
K_{T}^{l}=\sum_{e=1}^{n e} K_{e}
$$

where $n e$ is the number of structural elements and $K_{e}$ is the tangent stiffness matrix of the element with material nonlinearity. Differentiating Equation 
45 with respect to any generalized variable, $x_{j}$, sensitivity of the tangent stiffness matrix can be obtained from:

$$
\frac{d K_{T}^{(l)}}{d x_{j}}=\sum_{e=1}^{n e} \frac{d K_{e}}{d x_{j}}
$$

Based on the last equation, to compute tangent stiffness sensitivity, it is sufficient to calculate sensitivity of tangent stiffness of all elements. Sensitivity of tangent stiffness matrix of each element is obtained by differentiating from Equation 21:

$$
\frac{d K_{e}}{d x_{j}}=T_{e}^{T} \frac{d K_{t e}}{d x_{j}} T_{e}
$$

In the above equation, $\frac{d K_{t e}}{d x_{j}}$ is the sensitivity of the tangent stiffness matrix of the element including axial effects sensitivity and bending effects sensitivity in local system and can be obtained by differentiating from Equation 20:

$$
\frac{d K_{t e}}{d x_{j}}=\frac{d K_{a}}{d x_{j}}+\frac{d K_{b}}{d x_{j}}
$$

It is noted that the first and second terms in the right hand side of Equation 48 must be properly assembled. The first term represents the axial stiffness while the second one represents flexural stiffness. These two sensitivity matrix are obtained by differentiating Equations 22 and 23 noting that matrices of $K_{a 0}$ and $P_{E}$ utilized in these equations are independent of design variables and can be determined as follow:

$\frac{d K_{a}}{d x_{j}}=\frac{d A}{d x_{j}} K_{a 0}$

$$
\frac{d K_{b}}{d x_{j}}=R_{E} \frac{d K_{S}}{d x_{j}} R_{E}^{T}
$$

Assuming rigid diaphragm for floors, there will be no change in axial forces of beams. Accordingly, the axial stiffness sensitivity for beams is zero. For column elements by defining $A=b h$, the sensitivity of $A$ with respect to $b$ is $h$, and with respect to $h$ is $b$. It is zero with respect to other variables. The sensitivity of matrix $K_{S}$ utilized in Equation 50 is determined by differentiating from Equation 24:

$$
\frac{d K_{S}}{d x_{j}}=\frac{d \tilde{L}}{d x_{j}} K_{S}^{\prime} \tilde{L}^{-1}+\tilde{L} \frac{d K_{S}^{\prime}}{d x_{j}} \tilde{L}^{-1}+\tilde{L} K_{S}^{\prime} \frac{d \tilde{L}^{-1}}{d x_{j}}
$$

where:

$$
\left.\begin{array}{l}
\left.\frac{\tilde{d}}{d x_{j}}=-\frac{1}{1-\lambda_{A}-\lambda_{B}}\left\{\left(\frac{d \lambda_{A}}{d x_{j}}+\frac{d \lambda_{B}}{d x_{j}}\right) \tilde{L}+\left[\begin{array}{cc}
\frac{d \lambda_{B}}{d x_{j}} & -\frac{d \lambda_{A}}{d x_{j}} \\
-\frac{d \lambda_{B}}{d x_{j}} & \frac{d \lambda_{A}}{d x_{j}}
\end{array}\right]\right\}\right) \\
\frac{d \tilde{L}^{-1}}{d x_{j}}=-\left[\begin{array}{ll}
\frac{d \lambda_{A}}{d x_{j}} & \frac{d \lambda_{A}}{d x_{j}} \\
\frac{d \lambda_{B}}{d x_{j}} & \frac{d \lambda_{B}}{d x_{j}}
\end{array}\right]
\end{array}\right\}
$$

In Equation 52, sensitivity of $\lambda_{A}$ with respect to $h_{A}$ is $1 / 2 L$; also sensitivity of $\lambda_{B}$ with respect to $h_{B}$ is $1 / 2 L$. For a typical beam $h_{A}$ and $h_{B}$ have been shown in Figure 3. Similar definitions can be made for columns between two floor beams. In Equation 51 sensitivities of matrix $K_{S}^{\prime}$ with respect to any design variable can be obtained by differentiation of its elements as follow:

$$
\left.\begin{array}{l}
\frac{d K_{S 11}}{d x_{j}}=\frac{12 f_{b} L^{\prime}}{D_{e}}\left(\frac{d E I_{0}}{d x_{j}} E I_{A} E I_{B}+\frac{d E I_{A}}{d x_{j}} E I_{0} E I_{B}\right. \\
\left.+\frac{d E I_{B}}{d x_{j}} E I_{A} E I_{0}\right)+\frac{12 E I_{0} E I_{A} E I_{B}}{D_{e}}\left(\frac{d f_{b}}{d x_{j}} L^{\prime}\right. \\
\left.+\frac{d L^{\prime}}{d x_{j}} f_{b}-\frac{d D_{e}}{d x_{j}} \frac{L_{f_{b}}^{\prime}}{D_{e}}\right)
\end{array}\right\}
$$

$\frac{d K_{S 12}}{d x_{j}}=\frac{d K_{S 11}}{d x_{j}} \frac{f_{c}}{f_{b}}+\frac{d f_{c}}{d x_{j}} \frac{K_{S 11}}{f_{b}}-\frac{d f_{b}}{d x_{j}} \frac{f_{c} K_{S 11}}{f_{b}^{2}}$

$\frac{d K_{S 22}}{d x_{j}}=\frac{d K_{S 11}}{d x_{j}} \frac{f_{a}}{f_{b}}+\frac{d f_{a}}{d x_{j}} \frac{K_{S 11}}{f_{b}}-\frac{d f_{b}}{d x_{j}} \frac{f_{a} K_{S 11}}{f_{b}^{2}}$

Where

$$
\left.\begin{array}{l}
\frac{d f_{a}}{d x_{j}}=S_{2}^{\prime}\left(6 \alpha_{A}-4 \alpha_{A}^{2}+\alpha_{A}^{3}\right)+3 S_{3} \alpha_{B}^{2} \frac{d \alpha_{B}}{d x_{j}} \\
+S_{2}\left(6-8 \alpha_{A}+3 \alpha_{A}^{2}\right) \frac{d \alpha_{A}}{d x_{j}}+S_{3}^{\prime} \alpha_{B}^{3}+4 S_{1}^{\prime}
\end{array}\right\}
$$




$$
\left.\begin{array}{l}
\left.\begin{array}{l}
\frac{d f_{b}}{d x_{j}}=S_{3}^{\prime}\left(6 \alpha_{B}-4 \alpha_{B}^{2}+\alpha_{B}^{3}\right)+3 S_{2} \alpha_{A}^{2} \frac{d \alpha_{A}}{d x_{j}} \\
+S_{3}\left(6-8 \alpha_{B}+3 \alpha_{B}^{2}\right) \frac{d \alpha_{B}}{d x_{j}}+S_{2}^{\prime} \alpha_{A}^{3}+4 S_{1}^{\prime}
\end{array}\right\} \\
\left.\begin{array}{l}
\frac{d f_{c}}{d x_{j}}=-S_{2}^{\prime}\left(2 \alpha_{A}^{2}-\alpha_{A}^{3}\right)-S_{2}\left(4 \alpha_{A}-3 \alpha_{A}^{2}\right) \frac{d \alpha_{A}}{d x_{j}} \\
-S_{3}^{\prime}\left(2 \alpha_{B}^{2}-\alpha_{B}^{3}\right)-S_{3}\left(4 \alpha_{B}-3 \alpha_{B}^{2}\right) \frac{d \alpha_{B}}{d x_{j}}-2 S_{1}^{\prime}
\end{array}\right\} \\
\frac{d D_{e}}{d x_{j}}=\left(\frac{d f_{a}}{d x_{j}} f_{b}+\frac{d f_{b}}{d x_{j}} f_{a}-2 f_{c} \frac{d f_{c}}{d x_{j}}\right) L^{\prime 2} \\
+2\left(f_{a} f_{b}-f_{c}^{2}\right) L^{\prime} \frac{d L^{\prime}}{d x_{j}} \\
\frac{d L^{\prime}}{d x_{j}}=-\left(\frac{d \lambda_{a}}{d x_{j}}+\frac{d \lambda_{b}}{d x_{j}}\right) L
\end{array}\right\}
$$

where:

$$
\left.\begin{array}{l}
S_{1}^{\prime}=\frac{d E I_{A}}{d x_{j}} E I_{B}+\frac{d E I_{B}}{d x_{j}} E I_{A} \\
S_{2}^{\prime}=\left(\frac{d E I_{0}}{d x_{j}}-\frac{d E I_{A}}{d x_{j}}\right) E I_{B}+\left(E I_{0}-E I_{A}\right) \frac{d E I_{B}}{d x_{j}} \\
S_{3}^{\prime}=\left(\frac{d E I_{0}}{d x_{j}}-\frac{d E I_{B}}{d x_{j}}\right) E I_{A}+\left(E I_{0}-E I_{B}\right) \frac{d E I_{A}}{d x_{j}}
\end{array}\right\}
$$

In these equations, the derivative of $E I_{0}$ with respect to any variable $X$ can be obtained by differentiating from Equation 29 with respect to $\mathrm{X}$. This leads to Equation 62:

$$
\left.\begin{array}{l}
\frac{d E I_{0}}{d x_{j}}=\frac{2}{E I_{A 0}+E I_{B 0}}\left(\frac{d E I_{A 0}}{d x_{j}} E I_{B 0}+\frac{d E I_{B 0}}{d x_{j}} E I_{A 0}\right) \\
-\frac{2 E I_{A 0} E I_{B 0}}{\left(E I_{A 0}+E I_{B 0}\right)^{2}}\left(\frac{d E I_{A 0}}{d x_{j}}+\frac{d E I_{B 0}}{d x_{j}}\right)
\end{array}\right\}
$$

For an element with two end moments greater than cracking moment and single curvature, the sensitivity of $\mathrm{EI}_{0}$ with respect to $\mathrm{X}$ should be modified by Equation 63. Sensitivity of $E I_{A 0}$ and $E I_{B 0}$ will be obtained from Equation 64 considering the case of $M_{p} \leq M_{c r p}$ :

$$
\left.\begin{array}{l}
\frac{d E I_{0}}{d x_{j}}=\frac{2}{E I_{A}+E I_{B}}\left(\frac{d E I_{A}}{d x_{j}} E I_{B}+\frac{d E I_{B}}{d x_{j}} E I_{A}\right) \\
-\frac{2 E I_{A} E I_{B}}{\left(E I_{A}+E I_{B}\right)^{2}}\left(\frac{d E I_{A}}{d x_{j}}+\frac{d E I_{B}}{d x_{j}}\right)
\end{array}\right\}
$$

In Equations 53 and 61 to 63, to use the sensitivity $E I_{A}$ and $E I_{B}$, it is sufficient to differentiate Equations 17 to 19 with respect to $\mathrm{x}_{\mathrm{j}}$ as follow:

$$
\left.\begin{array}{l}
\frac{d E I_{p}}{d x_{j}}=\frac{1}{\phi_{c r p}} \frac{d M_{c r p}}{d x_{j}}-\frac{M_{c r p}}{\phi_{c r p}^{2}} \frac{d \phi_{c r p}}{d x_{j}} \\
\frac{d E I_{p}}{d x_{j}}=\frac{1}{\phi_{y p}-\phi_{c r p}}\left(\frac{d M_{y p}}{d x_{j}}-\frac{d M_{c r p}}{d x_{j}}\right) \\
-\frac{M_{y p}-M_{c r p}}{\left(\phi_{y p}-\phi_{c r p}\right)^{2}}\left(\frac{d \phi_{y p}}{d x_{j}}-\frac{d \phi_{c r p}}{d x_{j}}\right)
\end{array}\right\}
$$

$$
\left.\begin{array}{c}
\frac{d E I_{p}}{d x_{j}}=\frac{1}{\phi_{u p}-\phi_{y p}}\left(\frac{d M_{u p}}{d x_{j}}-\frac{d M_{y p}}{d x_{j}}\right) \\
-\frac{M_{u p}-M_{y p}}{\left(\phi_{u p}-\phi_{y p}\right)^{2}}\left(\frac{d \phi_{u p}}{d x_{j}}-\frac{d \phi_{y p}}{d x_{j}}\right)
\end{array}\right\}
$$

where Equations 64, 65 and 66 are used for zones of 1, 2 and 3 of $M-\phi$ curve, respectively.

As observed above, sensitivities of momentcurvature characteristics in various states of cracking, yielding and ultimate state have been appeared in three last equations. To calculate these sensitivities, both axial and flexural effects are considered is our next problem, because existence of axial force causes to change them. Since axial forces are variative at various load steps, momentcurvature characteristics are not the same at all load steps. Hence, their sensitivities should be determined by differentiating from Equations 1 to (6) at each load step. These sensitivity calculations have been reported in the next section. According to section 2.2, the sensitivity of $\alpha_{A}$ and $\alpha_{B}$ that have been used in Equations 56 to 58, are zero provided that moments are less than cracking moments or the element undergoes single curvature with end moments greater than cracking moment. For other cases the sensitivities can be 
determined by differentiating from Equation 28:

$$
\left.\begin{array}{l}
\frac{d \alpha_{p}}{d x_{j}}=\frac{1}{\left|M_{A}-M_{B}\right|}\left[\left(\frac{d M_{p}}{d x_{j}} \frac{M_{p}}{\left|M_{p}\right|}-\frac{d M_{c r p}}{d x_{j}}\right)\right. \\
\left.-\frac{\left|M_{p}\right|-M_{c r p}}{\left(M_{A}-M_{B}\right)}\left(\frac{d M_{A}}{d x_{j}}-\frac{d M_{B}}{d x_{j}}\right)\right]
\end{array}\right\}
$$

where $d M_{p} / d x_{j}$ is the sensitivity of moment at end ' $\mathrm{p}$ ' of the element; its value will be presented in section 3.3. In Equation 67, if $\alpha_{p}>1$, its sensitivity is zero and if $\alpha_{p}<\alpha_{p m}$, its sensitivity is equal to sensitivity of $\alpha_{p m}$. When $\alpha_{A}+\alpha_{B}>1$, the sensitivity of $E I_{0}, \alpha_{A}$ and $\alpha_{B}$ must be modified by differentiation of Equations 31 to 33:

$$
\begin{gathered}
\frac{d \alpha_{A}^{\prime}}{d x_{j}}=\frac{f_{A x}-f_{B x}+\frac{f_{B x}-f_{0 x}}{\alpha_{B}}-\frac{d \alpha_{B}}{d x_{j}} \frac{f_{B}-f_{0}}{\alpha_{B}^{2}}}{\frac{f_{A}-f_{0}}{\alpha_{A}}+\frac{f_{B}-f_{0}}{\alpha_{B}}} \\
-\left(\frac{f_{A x}-f_{0 x}}{\alpha_{A}}-\frac{d \alpha_{A}}{d x_{j}} \frac{f_{A}-f_{0}}{\alpha_{A}^{2}}+\frac{f_{B x}-f_{0 x}}{\alpha_{B}}\right. \\
\left.-\frac{d \alpha_{B}}{d x_{j}} \frac{f_{B}-f_{0}}{\alpha_{B}^{2}}\right) \frac{f_{A}-f_{B}+\frac{f_{B}-f_{0}}{\alpha_{B}}}{\left(\frac{f_{A}-f_{0}}{\alpha_{A}}+\frac{f_{B}-f_{0}}{\alpha_{B}}\right)^{2}}
\end{gathered}
$$

$\frac{d \alpha_{B}^{\prime}}{d x_{j}}=-\frac{d \alpha_{A}^{\prime}}{d x_{j}}$

$\frac{d f_{0}^{\prime}}{d x_{j}}=f_{A x}-\frac{f_{A x}-f_{0 x}}{\alpha_{A}} \alpha_{A}^{\prime}$

$\left.-\left(f_{A}-f_{0}\right)\left(\frac{1}{\alpha_{A}} \frac{d \alpha_{A}^{\prime}}{d x_{j}}-\frac{\alpha_{A}^{\prime}}{\alpha_{A}^{2}} \frac{d \alpha_{A}}{d x_{j}}\right)\right\}$

where:

$$
f_{A x}=-\frac{d E I_{A}}{d x_{j}} \frac{1}{E I_{A}^{2}} ; f_{B x}=-\frac{d E I_{B}}{d x_{j}} \frac{1}{E I_{B}^{2}} ; f_{0 x}=-\frac{d E b_{0}}{d x_{j}} \frac{1}{E I_{0}^{2}}
$$

3.1.2. Sensitivity of loads Since seismic loads depend on the natural period of the structure and the natural period depends on structural stiffness and building mass, any change in the structure stiffness causes a change in the external loads. Since gravity loads are constant, the sensitivity of external load vector at any load step $l$ comprises only sensitivity of lateral loads.

$\frac{d P^{l}}{d x_{j}}=\frac{d P_{E}^{l-1}}{d x_{j}}+\frac{d \Delta P_{E}}{d x_{j}}$

where the second term in right hand side is the sensitivity of incremental seismic loads and is obtained from

$\frac{d \Delta P_{E}}{d x_{j}}=\Delta V_{b} \cdot \frac{d C_{v}}{d x_{j}}$

where the components of $d C_{v} / d x_{j}$ vector are found from the following equation:

$\frac{d c_{v, s}}{d x_{j}}=\frac{W_{s} H_{s}^{k}}{\sum_{s=1}^{n s} W_{s} H_{s}^{k}}\left(l_{n} H_{s}-\frac{\sum_{s=1}^{n s} W_{s} H_{s}^{k} l_{n} H_{s}}{\sum_{s=1}^{n s} W_{s} H_{s}^{k}}\right) \frac{d k}{d x_{j}}$

In the above equation, for $T \leq 0.5$ and $T \geq 2.5$, the sensitivity of $k$ is zero. For other values of $T$, derivative of Equation 37 gives:

$d k / d x_{j}=0.5 d T / d x_{j}$

where $d T / d x_{j}$ is the sensitivity of the fundamental period of the structure and can be found from the following relation [20]:

$\frac{d T}{d x_{j}}=-\frac{T^{3}}{8 \pi^{2}} \phi^{T} \frac{d K}{d x_{j}} \phi$

where $\phi$ is the first mode shape of the structure.

3.2. Force Sensitivity Since in nonlinear structures, displacement is an implicit function of internal forces; force sensitivities need to be calculated for computation of displacement sensitivity. Sensitivity of the incremental internal forces of any typical element at load step $l$ can be determined by differentiating from Equation 40:

$$
\frac{d \Delta F_{e}^{l}}{d x_{j}}=\frac{d K_{e}^{l}}{d x_{j}} \Delta u_{e}^{l}+K_{e}^{l} \frac{d \Delta u_{e}^{l}}{d x_{j}}
$$


The sensitivity of internal forces of the element is updated by Equation 78:

$$
\frac{d F_{e}^{l}}{d x_{j}}=\frac{d F_{e}^{l-1}}{d x_{j}}+\frac{d \Delta F_{e}^{l}}{d x_{j}}
$$

where $\frac{d F_{e}^{l-1}}{d x_{j}}$ is the sensitivity vector of internal forces at previous load step and $\frac{d \Delta F_{e}^{l}}{d x_{j}}$ is the sensitivity vector of incremental internal forces determined from Equation 77. Components of the sensitivity vector, which is obtained by Equation 78 , give sensitivity of forces such as moment sensitivity and axial force sensitivity which are appeared in some sensitivity equations derived in previous sections.

3.3. Moment-Curvature Curve Sensitivity Since moment-curvature relations are functions of design variables and axial forces and sensitivity equations derived in previous sections are dependent on their sensitivities, these sensitivities including cracking moment sensitivity, yielding moment sensitivity, ultimate moment sensitivity, cracking curvature sensitivity, yielding curvature sensitivity and ultimate curvature sensitivity need to be determined. These sensitivities at each load step are variable for existence of axial forces. This causes that nonlinear sensitivity analysis be more difficult than the state in which, sensitivity of axial loads is ignored. Sensitivities of moment-curvature characteristics can be obtained by differentiating from Equations 1 to 6 at various states:

(a) Cracking state

$$
\begin{aligned}
& \frac{d M_{c r}}{d x_{j}}=\frac{f_{r}\left(d I / d x_{j}\right)}{d h / d x_{j}-d y / d x_{j}}-\frac{f_{r} I\left(d h / d x_{j}-d y / d x_{j}\right)}{(h-y)^{2}} \\
& +\frac{N\left(d d / d x_{j}\right)}{6}+\frac{d\left(d N / d x_{j}\right)}{6} \\
& \frac{d \varphi_{c r}}{d x_{j}}=\frac{f_{r}\left(d y / d x_{j}-d h / d x_{j}\right)}{E_{c}(h-y)^{2}}
\end{aligned}
$$

(b) Yielding state

$$
\begin{aligned}
& \frac{d M_{y}}{d x_{j}}=0.5 f_{c}\left(\frac{d b^{*}}{d x_{j}} d^{2}+2 b^{*} d \frac{d d}{d x_{j}}\right)\left[(1+\beta-\eta) n_{0}+(2-\eta) p\right. \\
& \left.+(\eta-2 \beta) \alpha p^{\prime}\right]+0.5 f_{c} b^{*} d^{2}\left[(1+\beta-\eta) \frac{d n_{0}}{d x_{j}}+\left(\frac{d \beta}{d x_{j}}-\frac{d \eta}{d x_{j}}\right) n_{0}\right. \\
& \left.+(2-\eta) \frac{d p}{d x_{j}}-p \frac{d \eta}{d x_{j}}\right]+\left(\frac{d \eta}{d x_{j}}-2 \frac{d \beta}{d x_{j}}\right) \alpha p^{\prime}+(\eta-2 \beta)\left(\alpha \frac{d p^{\prime}}{d x_{j}}+p^{\prime} \frac{d \alpha}{d x_{j}}\right)
\end{aligned}
$$

$$
\frac{d \phi_{y}}{d x_{j}}=\frac{\left(d c / d x_{j}\right)(1-k) d+\left(d k / d x_{j}\right) d c-\left(d d / d x_{j}\right)(1-k) c}{(1-k)^{2} d^{2}} \varepsilon_{y}
$$

(c) Ultimate state

$$
\frac{d M_{u}}{d x_{j}}=\left(1.24-0.15 p-0.5 n_{0}\right) \frac{d M_{y}}{d x_{j}}-\left(0.15 \frac{d p}{d x_{j}}+0.5 \frac{d n_{0}}{d x_{j}}\right) M_{y}
$$

$$
\frac{d \phi_{u}}{d x_{j}}=\frac{\left[\left(R^{2}+S\right)^{0.5}-R\right] \frac{d c}{d x_{j}}-c\left[0.5\left(2 R \frac{d R}{d x_{j}}+\frac{d S}{d x_{j}}\right)\left(R^{2}+S\right)^{-0.5}-\frac{d R}{d x_{j}}\right]}{\left[\left(R^{2}+S\right)^{0.5}-R\right]^{2}} \beta_{1} \varepsilon_{u}
$$

In above equations, unknown sensitivities are obtained by differentiating from Equations 7 to 16 and $\mathrm{R}$ aqnd $\mathrm{S}$ (the extended form should be used) equations with respect to design variable $x_{j}$ from the following equations:

$$
\begin{aligned}
& \frac{d I}{d x_{j}}=\frac{d b_{t}}{d x_{j}} \frac{t^{3}}{12}+\frac{d t}{d x_{j}} b_{t} t^{2}+\frac{d b_{t}}{d x_{j}} t\left(y-\frac{t}{2}\right)^{2}+b_{t} \frac{d t}{d x_{j}}\left(y-\frac{t}{2}\right)^{2} \\
& +2 b_{t} t\left(y-\frac{t}{2}\right)\left(\frac{d y}{d x_{j}}-\frac{1}{2} \frac{d t}{d x_{j}}\right)+\frac{d b_{b}}{d x_{j}} \frac{(h-t)^{3}}{12}+\frac{b_{b}}{4}(h-t)^{2} \\
& \times\left(\frac{d h}{d x_{j}}-\frac{d t}{d x_{j}}\right)+\left[\frac{d b_{b}}{d x_{j}}(h-t)+b_{b}\left(\frac{d h}{d x_{j}}-\frac{d t}{d x_{j}}\right)\right]\left(\frac{h+t}{2}-y\right)^{2} \\
& +2 b_{b}(h-t)\left(\frac{1}{2} \frac{d h}{d x_{j}}+\frac{1}{2} \frac{d t}{d x_{j}}-\frac{d y}{d x_{j}}\right)\left(\frac{h+t}{2}-y\right)+\left(n_{s c}-1\right) \\
& \times\left[\frac{d A_{s}}{d x_{j}}(d-y)^{2}+\frac{d A_{s}^{\prime}}{d x_{j}}\left(y-d^{\prime}\right)^{2}\right]+2\left(n_{s c}-1\right)\left[\frac{d y}{d x_{j}} A_{s}^{\prime}\left(y-d^{\prime}\right)\right. \\
& \left.+A_{s}(d-y)\left(\frac{d h}{d x_{j}}-\frac{d y}{d x_{j}}\right)\right]
\end{aligned}
$$

IJE Transactions A: Basics 
$\frac{d \eta}{d x_{j}}=0.525 \frac{\left(\phi_{y} d-\varepsilon_{y}\right)^{-0.3}}{\left(1+\varepsilon_{y} / \varepsilon_{0}\right) \varepsilon_{0}^{0.7}}\left(d \frac{d \phi_{y}}{d x_{j}}+\phi_{y} \frac{d h}{d x_{j}}\right)$
$\frac{d \alpha}{d x_{j}}=-\frac{d \beta}{d x_{j}} \frac{\left(\phi_{y} d-\varepsilon_{y}\right)}{\varepsilon_{y}}+\frac{(1-\beta)}{\varepsilon_{y}}\left(d \frac{d \phi_{y}}{d x_{j}}+\phi_{y} \frac{d h}{d x_{j}}\right)-\frac{d \beta}{d x_{j}}$

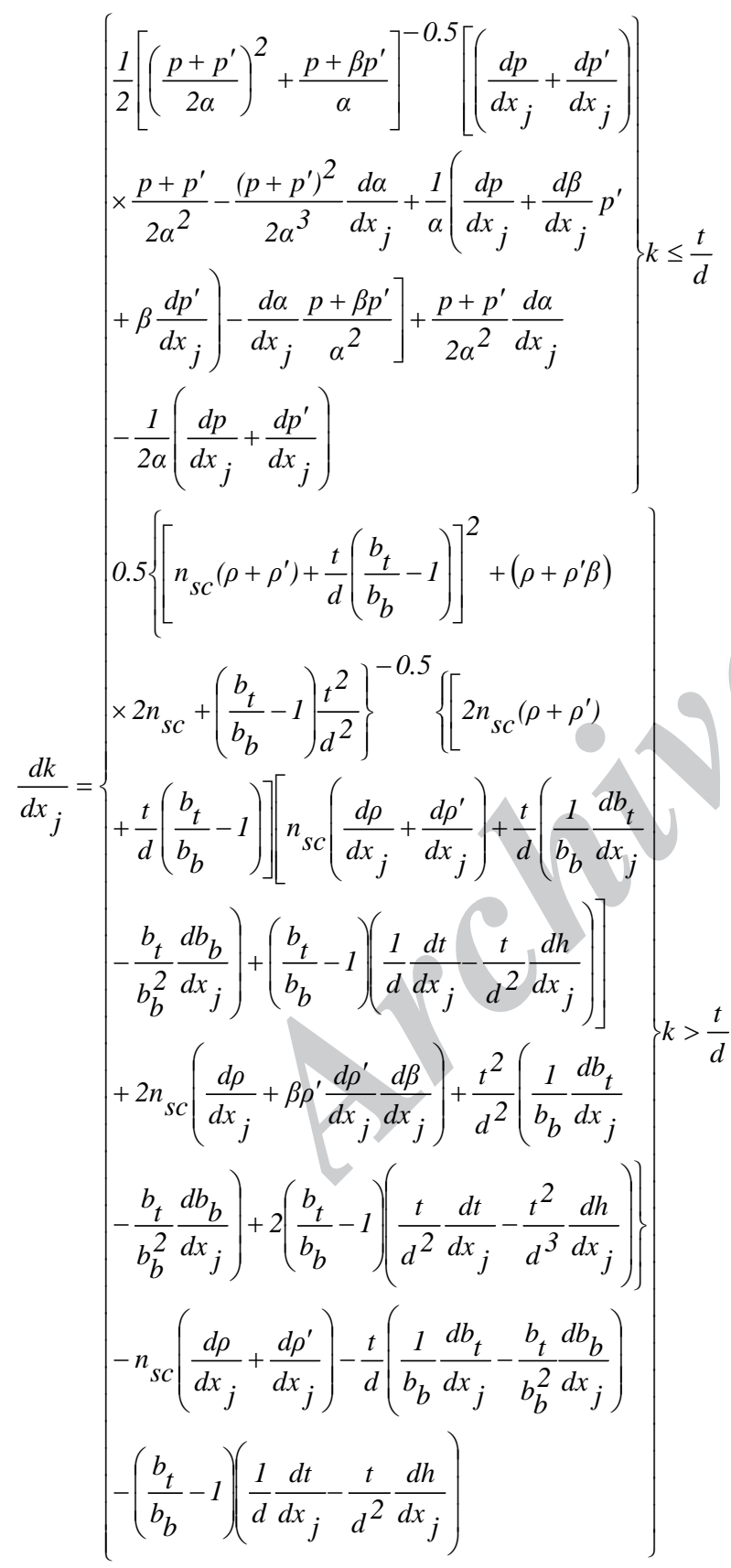

(88) $\frac{d y}{d x_{j}}=\frac{1}{\left[0.5 b_{t} t^{2}+0.5 b_{b}\left(h^{2}-t^{2}\right)+\left(n_{s c}-1\right)\left(A_{s} d+A_{s}^{\prime} c\right)\right]^{2}}$

$\times\left\{\left[0.5 \frac{d b_{t}}{d x_{j}} t^{2}+b_{t} t \frac{d t}{d x_{j}}+0.5 \frac{d b_{b}}{d x_{j}}\left(h^{2}-t^{2}\right)+b_{b}\left(h \frac{d h}{d x_{j}}\right.\right.\right.$

$\left.-t \frac{d t}{d x_{j}}\right)+\left(n_{s c}-1\right)\left(\frac{d A_{s}}{d x_{j}} d+A_{s} \frac{d h}{d x_{j}}+\frac{d A_{s}^{\prime}}{d x_{j}} d^{\prime}\right)\left[b_{b}(h-t)\right.$

$\left.+b_{t} t+\left(n_{s c}-1\right)\left(A_{s}+A_{s}^{\prime}\right)\right]-\left[\frac{d b_{t}}{d x_{j}} t+b_{t} \frac{d t}{d x_{j}}+\frac{d b_{b}}{d x_{j}}(h-t)\right.$

$\left.+b_{b} \frac{d h}{d x_{j}}+\left(n_{s c}-1\right)\left(\frac{d A_{s}}{d x_{j}}+\frac{d A_{s}^{\prime}}{d x_{j}}\right)\right]\left[0.5 b_{t} t^{2}+\left(h^{2}-t^{2}\right)\right.$

$\left.\times 0.5 b_{b}+\left(n_{s c}-1\right)\left(A_{s} d+A_{s}^{\prime} c\right)\right]$

$\frac{d \rho}{d x_{j}}=\frac{1}{b_{b} d} \frac{d A_{s}}{d x_{j}}-\frac{A_{s}}{b_{b}^{2} d^{2}}\left(\frac{d b_{b}}{d x_{j}} d+b_{b} \frac{d h}{d x_{j}}\right)$

$\frac{d \rho^{\prime}}{d x_{j}}=\frac{1}{b_{b} d} \frac{d A_{s}^{\prime}}{d x_{j}}-\frac{A_{s}^{\prime}}{b_{b}^{2} d^{2}}\left(\frac{d b_{b}}{d x_{j}} d+b_{b} \frac{d h}{d x_{j}}\right)$

$\frac{d p}{d x_{j}}=\frac{f_{y}}{f_{c}} \frac{d \rho}{d x_{j}}$

$\frac{d p^{\prime}}{d x_{j}}=\frac{f_{y}}{f_{c}} \frac{d \rho^{\prime}}{d x_{j}}$

$\frac{d \beta}{d x_{j}}=-\frac{d^{\prime}}{d^{2}} \frac{d h}{d x_{j}}$

$\frac{d c}{d x_{j}}=-\frac{1.5 n_{0}}{(0.84+p)^{2}} \frac{d p}{d x_{j}}+\left(\frac{0.45}{0.84+p}-0.05\right) \frac{1}{0.3} \frac{d n_{0}}{d x_{j}}$

$\frac{d n_{0}}{d x_{j}}=\frac{1}{f_{c} b d} \frac{d N}{d x_{j}}-\frac{N}{f_{c} b^{2} d^{2}}\left(\frac{d b}{d x_{j}} d+b \frac{d h}{d x_{j}}\right)$

$\frac{d R}{d x_{j}}=\frac{1}{1.7 f_{c}}\left[\left(\frac{d \rho^{\prime}}{d x_{j}} \varepsilon_{u} E_{s}-\frac{d \rho}{d x_{j}} f_{y}\right) d+\left(\rho^{\prime} \varepsilon_{u} E_{s}-\rho_{y}\right) \frac{d h}{d x_{j}}\right](97)$ 
$\frac{d S}{d x_{j}}=\frac{\varepsilon_{u} E_{s} \beta_{1} d^{\prime}}{0.85 f_{c}}\left(\frac{d \rho^{\prime}}{d x_{j}} d+\rho^{\prime} \frac{d h}{d x_{j}}\right)$

In equations presented in this section, values of $\frac{d b_{t}}{d x_{j}}, \frac{d b_{b}}{d x_{j}}, \frac{d t}{d x_{j}}$ and $\frac{d h}{d x_{j}}$ are equal to 1 for $x_{j}=b_{t}, b_{b}, t$ and $h$, respectively. Values of these parameters for other design variables are equal to zero. Axial load sensitivity $\left(\frac{d N}{d x_{j}}\right)$ that exists in some equations is one component of sensitivity vector obtained from section 3.2.

\subsection{Summary of Sensitivity Analysis Procedure}

The nonlinear sensitivity analysis of RC frames that was developed in this study can be carried out in the following steps:

1. Compute the first mode shape, first period and its sensitivity from Equation 76

2. Calculate the matrixes $\tilde{L}$ and $\tilde{L}^{-1}$ and their derivatives for each element from Equations 25 and 52

3. Compute the lateral load distribution factors and their sensitivities by Equations 36 and 74

4 Set load step index $l=1$ and apply the gravity loads only

5. Determine the moment-curvature relations from Equations 1 to 6 and compute their derivatives from section 3.3

6. Compute the lateral loads and their sensitivities from Equations 35 and 73

7. Compute the flexural stiffness and yield penetration parameters and their sensitivities for each element from Equations 17 to 19 and Equations 64 to 66

8. Compute the yield penetration parameters and their sensitivities for each element from Equations 28, 31, 32, and Equations 67 to 69

9. Compute the tangent stiffness matrix and its sensitivity for each element by Equations 21 and 47

10. Assemble the global tangent stiffness matrix and its matrix of sensitivity by Equations 45 and 46

11. Solve Equation 38 for displacements and find their incremental sensitivities from Equation 43
12. Calculate the internal forces and their sensitivities from Equations 40 and 77

13. Set $l=l+1$, if the roof lateral displacement is greater than target displacement, stop the sensitivity analysis and go to step 14; otherwise go to step 5 .

14. Compute the total incremental sensitivities coefficients

\section{NUMERICAL EXAMPLE}

A three-story, two-bay planar frame of Figure 4 is used to illustrate the method of proposed analytical nonlinear sensitivity analysis. The concrete is assumed to have a cylinder strength of $30 \mathrm{MPa}$, a modulus of rupture of $3.45 \mathrm{MPa}$, a modulus of elasticity of $27,400 \mathrm{MPa}$, a strain of 0.002 (unit?) at maximum strength and an ultimate strain of 0.003 . The steel has yield strength of $300 \mathrm{MPa}$ and a modulus of elasticity of $200,000 \mathrm{MPa}$. A uniformly distributed gravity load of $12 \mathrm{KN} / \mathrm{m}$ is applied on the beams of each story. Reinforcements have the cover to the steel centroid of $50 \mathrm{~mm}$. It is assumed that columns and beams have rectangular cross sections. Fourteen design variables which have been given in Table 1 are defined in this frame. In order to allow the structure to enter to the inelastic behavior, target displacement of $2 \%$ has been chosen as a stop criterion for load steps of sensitivity analysis.

The pushover (capacity) curve of this frame is shown in Figure 5 and target point has specified on it for two cases namely (i) flexural effect only, C0 and (ii) both flexural and axial effects, $\mathrm{C} 1$. Nonlinear sensitivity analysis is performed on this frame by the proposed Analytical Method (AM)

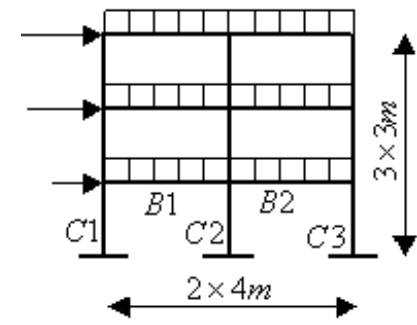

Figure 4. A three-story, two-bay RC frame. 
TABLE 1. Design variables.

\begin{tabular}{ccc}
\hline $\begin{array}{c}\text { Number of } \\
\text { Design Variable }\end{array}$ & Description & Value \\
\hline 1 & Width of columns C1 and C3 & $350 \mathrm{~mm}$ \\
2 & Height of columns C1 and C3 & $350 \mathrm{~mm}$ \\
3 & Width of columns C2 & $450 \mathrm{~mm}$ \\
4 & Height of columns C2 & $450 \mathrm{~mm}$ \\
5 & Reinforcement area of columns C1 and C3 on each face of column & $942.5 \mathrm{~mm}^{2}$ \\
6 & Reinforcement area of columns C2 on each face of column & $1570.8 \mathrm{~mm}^{2}$ \\
7 & Width of beams & $250 \mathrm{~mm}^{2}$ \\
8 & Height of beams & $350 \mathrm{~mm}^{2}$ \\
9 & Lower reinforcement area for beams B1 at left hand and for beams & $1256.6 \mathrm{~mm}^{2}$ \\
10 & B2 at right hand of beam (first and second stories) & \\
& Upper reinforcement area for beams B1 at left hand and for beams B2 \\
11 & at right hand of beam (first and second stories) & $942.5 \mathrm{~mm}^{2}$ \\
12 & Lower reinforcement area for beams B1 at right hand and for beams & \\
13 & B2 at left hand of beam (first and second stories) & $1256.6 \mathrm{~mm}^{2}$ \\
14 & Upper reinforcement area for beams B1 at right hand and for beams & $1570.8 \mathrm{~mm}^{2}$ \\
& B2 at left hand of beam (first and second stories) & $628.3 \mathrm{~mm}^{2}$ \\
\hline & Lower reinforcement area for third story beams & $628.3 \mathrm{~mm}^{2}$ \\
\hline
\end{tabular}

for cases of (i) and (ii) and compared to the results from central Finite Difference Method (FDM). As a sample, sensitivity analysis of the overall drift that may indirectly represent the sensitivity of internal forces to change in some design variables is considered. The results have been briefed in Table 2. For interpreting the results, for example, consider the design variable No.7. The overall drift sensitivity shows that its value will be decreased by $1.1499 \%$ and $-1.3875 \%$ for a unit change in the design variable No.7 for cases $i$ and ii, respectively. Values of nonlinear sensitivity coefficients predict that if the value of width of beams increases from $250 \mathrm{~mm}$ to $260 \mathrm{~mm}$; overall drift decreases $2 \%$ to $1.9885 \%$ and $1.9861 \%$ for C0 and $\mathrm{C} 1$, respectively. This prediction is very important in optimal performance-based design process. Sensitivity results for cases (i) and (ii) show that sensitivity coefficients of $\mathrm{C} 0$ and $\mathrm{C} 1$ are not the same. This difference is considerable for some design variables such as variable No.1. Therefore, existence of axial forces can affect sensitivity coefficients with respect to the design variables. For the present example, it is observed that the values of sensitivity coefficients of $\mathrm{C} 1$ are smaller than those of $\mathrm{C} 0$ for most of design variables. That is existence of axial loads decreases the displacement sensitivity in most of cases. In Table 2, percentage of error of FDM compared to $\mathrm{AM}$ has been evaluated from:

$$
\text { ERROR }=100\left(S_{F D M}-S_{A M}\right) / S_{A M}
$$

In Equation 99, $S_{F D M}$ and $S_{A M}$ are the sensitivity coefficients obtained from FDM and AM, respectively. In the FDM method, the design variables perturbation has been considered to be $1 \%$ and $0.001 \%$. Comparing the AM results with 


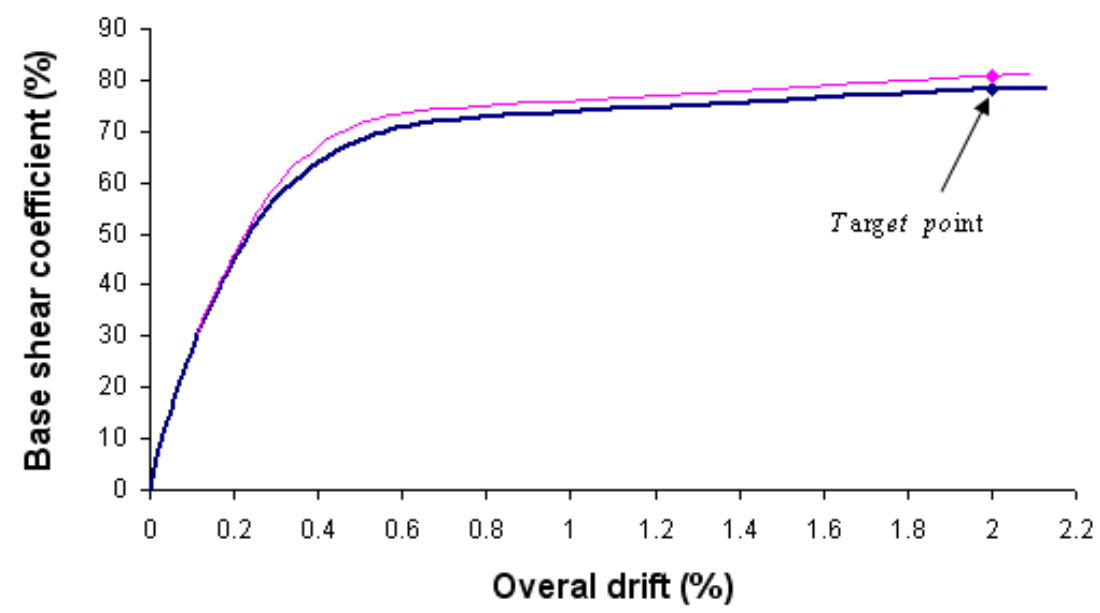

Figure 5. Capacity curve of the three-story RCMRF.

TABLE 2. Sensitivity Coefficients of Overall drift at Target Point (\%).

\begin{tabular}{ccccccccc}
\hline \multirow{2}{*}{ Method } & \multicolumn{7}{c}{ Sensitivity with Respect to Design Variable } \\
\cline { 2 - 8 } & & 1 & 4 & 5 & 7 & 8 & 9 & 12 \\
\cline { 2 - 9 } AM & $\mathrm{C} 0$ & -0.2688 & -10.8864 & -3284.1 & -1.1499 & -46.3542 & -2333.1 & -2183.6 \\
& $\mathrm{C} 1$ & -0.0064 & -7.9053 & -1642.1 & -1.3875 & -41.7281 & -1988.7 & -1993.5 \\
\hline \multirow{2}{*}{ FDM 1\% } & $\mathrm{C} 0$ & -0.4757 & -10.6962 & -3171.2 & -1.7722 & -61.3068 & -2140.8 & -2548.7 \\
& $\mathrm{C} 1$ & -2.7673 & -9.5303 & -2128.5 & -0.0931 & -56.8434 & -3020.5 & -3106.0 \\
\hline \multirow{2}{*}{ FDM 0.001\% } & $\mathrm{C} 0$ & -0.2688 & -10.8859 & -3284.1 & -1.1499 & -46.3487 & -2332.9 & -2183.3 \\
& $\mathrm{C} 1$ & -0.0064 & -7.9054 & -1641.9 & -1.3871 & -41.7283 & -1988.6 & -1993.4 \\
\hline \multirow{2}{*}{ ERROR 1\% } & $\mathrm{C} 0$ & 76.95 & -1.75 & -3.44 & 54.12 & 32.26 & -8.24 & 16.72 \\
& $\mathrm{C} 1$ & 42873 & 20.55 & 29.62 & 93.29 & 36.22 & 51.88 & 55.80 \\
\hline \multirow{2}{*}{ ERROR 0.001\% } & $\mathrm{C} 0$ & -0.03 & -0.005 & -0.001 & -0.001 & -0.01 & -0.006 & -0.01 \\
& $\mathrm{C} 1$ & 0.43 & 0.00 & 0.01 & 0.02 & 0.00 & 0.01 & 0.01 \\
\hline
\end{tabular}

that of FDM shows that by reducing perturbation values from $1 \%$ to $0.001 \%$ results of FDM for almost all design variables converge to AM results; that is, the AM results are in a good agreement with FDM results in very small perturbation cases. This matter verifies the AM results.

The results of sensitivity analysis by FDM shows that assuming perturbation of $1 \%$ in design variables, this method in all cases generates inaccurate results; while this is not the case in the linear sensitivity analysis. On the other hand, considering small perturbations for some design variables may lead to run-time errors. Hence, the proposed method can efficiently reduce errors of FDM. 


\section{CONCLUSION}

In the present study a procedure for exact nonlinear sensitivity analysis of RCMRF was proposed. The procedure includes both flexural and axial effects in the context of pushover analysis. The proposed sensitivity method does not face the difficulties that the FDM method confronts and can reduce errors of FDM.

Sensitivity analysis of a three-story RC frame illustrated the capability and effectiveness of the derived formulations. It was shown that existence of axial forces can affect sensitivity coefficients with respect to the design variables. Results of the case study for a nonlinear structure indicate that sensitivity calculation via FDM method may end up to inaccurate sensitivity results especially if the value of perturbation is high.

\section{REFERENCES}

1. Ryu, Y.S, Haririan, M., Wu, C.C., and Arora, J.S., "Structural design sensitivity analysis of nonlinear response", Computers and Structures, Vol. 21, (1985), 245-255.

2. Choi, K.K., and Santos, J.L.T., "Design sensitivity analysis of nonlinear structural systems Part I: Theory", International Journal for Numerical Methods in Engineering, Vol. 24, (1987), 2039-2055.

3. Gopalakrishna, H.S., and Greimann, F., "NewtonRaphson procedure for the sensitivity analysis of nonlinear structural behavior", Computers and Structures, Vol. 30, (1988), 1263-1273.

4. Santos, J.L.T, and Choi, K.K., "Shape design sensitivity analysis of nonlinear structural systems", Structural and Multidisciplinary Optimization, Vol. 4, (1992), 2335 .

5. Ohsaki, M., and Arora, J.S., "Design sensitivity analysis of elasto-plastic structures", International Journal for Numerical Methods in Engineering, Vol. 37, (1994), 737-762.

6. Lee, T.H., and Arora, J.S., "A computational method for design sensitivity analysis of elastoplastic Structures", Computer Methods in Applied Mechanics and Engineering, Vol. 122, (1995), 27-50.
7. Barthold, F.J., and Stein, E., "A continuum mechanicalbased formulation of the variational sensitivity analysis in structural optimization Part I: Analysis", Structural and Multidisciplinary Optimization, Vol. 11, (1996), 29-42.

8. Szewczyk, Z.P., and Ahmed, K.N., "A hybrid numerical/-neurocomputing strategy for sensitivity analysis of non-linear structures", Computers and Structures, Vol. 65, (1997), 869-880.

9. Yamazaki, K., "Sensitivity analysis of nonlinear material and its application to shape optimization", AIAA Journal, Vol. 36, (1998), 1113-1115.

10. Bugeda, G., Gil, L., and Onate, E., "Structural shape sensitivity analysis for nonlinear material models with strain softening", Structural and Multidisciplinary Optimization, Vol. 17, (1999), 162-171.

11. Schwarz, S., and Ramm, E., "Sensitivity analysis and optimization for nonlinear structural response", Engineering Computations, Vol. 18, (2001), 610.

12. Gong, Y., Xu, L., and Grierson, D.E., "Sensitivity analysis of steel moment frames accounting for geometric and material nonlinearity", Computers and Structures, Vol. 84, (2006), 462-475.

13. Habibi, A.R., and Moharrami, H., "Nonlinear Sensitivity Analysis of Reinforced Concrete Frames", Finite Elements in Analysis and Design, Vol. 46, (2010), 571-584.

14. FEMA-273, "NEHRP Guideline for the seismic rehabilitation of buildings", Federal Emergency Management Agency, Washington, DC, U.S.A., (1997).

15. ATC-40, "Seismic evaluation and retrofit of concrete buildings", Applied Technology Council, California Seismic Safety Commission, U.S.A., (1997).

16. Park, R., and Paulay, T., "Reinforced concrete structures", John Wiley and Sons, Inc., New York, U.S.A., (1974).

17. Park, Y.J., and Ang A.H.S., "Mechanistic seismic damage model for reinforced concrete", Journal of Structural Engineering, ASCE, Vol. 111, (1985), 722739

18. Park, Y.J., Reinhorn, A.M., and Kunnath, S.K., "IDARC: Inelastic damage analysis of frame shear-wall Structures", Technical report NCEER-87-0008, National center for earthquake engineering research, State University of New York at Buffalo, U.S.A., (1987)

19. Bathe. K.J., "Finite element procedures", Prentice Hall Inc., Englewood Cliff, New Jersey, U.S.A., (1996).

20. Moharrami, H., Alavinasab, S.A., "Design optimization of seismic resistant steel frames", Proceeding of the Eighth International Conference on Civil and Structural Engineering computing, Eisenstadt, Vienna, Austria, (2001). 


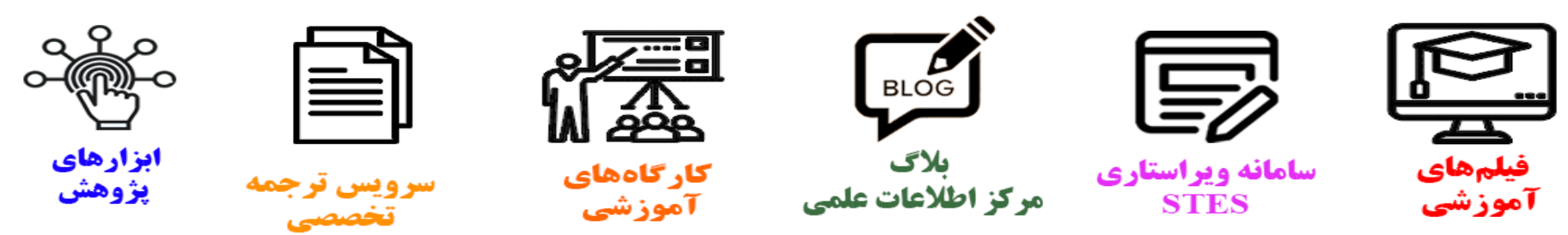

\section{(c)}

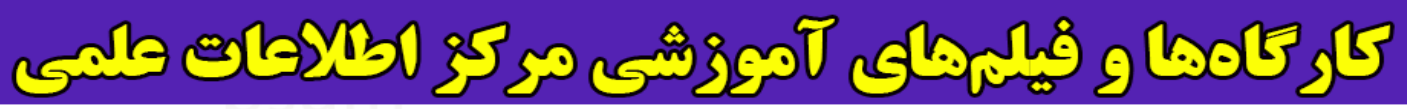
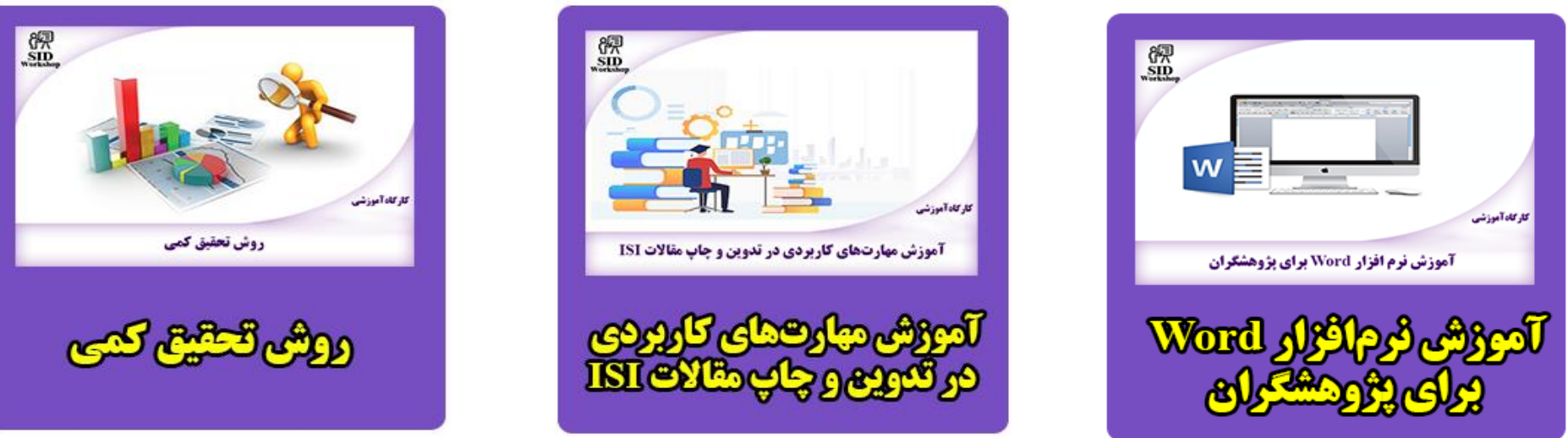